\title{
Distributed Output Regulation for a Class of Nonlinear Multi-Agent Systems with Unknown-Input Leaders *
}

\author{
Yutao Tang $^{\text {a }}$, Yiguang Hong ${ }^{\mathrm{b}}$, Xinghu Wang ${ }^{\mathrm{c}}$ \\ ${ }^{a}$ School of Automation, Beijing University of Posts and Telecommunications, Beijing 100876, China \\ ${ }^{\mathrm{b}}$ Key Laboratory of Systems and Control, Institute of Systems Science, Chinese Academy of Sciences, Beijing 100190, China \\ ${ }^{\mathrm{c}}$ Department of Automation, University of Science and Technology of China, Hefei 230027, China
}

\begin{abstract}
In this paper, a distributed output regulation problem is formulated for a class of uncertain nonlinear multi-agent systems subject to local disturbances. The formulation is given to study a leader-following problem when the leader contains unknown inputs and its dynamics is different from those of the followers. Based on the conventional output regulation assumptions and graph theory, distributed feedback controllers are constructed to make the agents globally or semi-globally follow the uncertain leader even when the bound of the leader's inputs is unknown to the followers.
\end{abstract}

Key words: Multi-agent systems, distributed output regulation, nonlinear dynamics, internal model

\section{Introduction}

The past decade has witnessed a rapid development in the field of multi-agent systems and fruitful results have been achieved for the leader-following coordination problem. In recent years, distributed output regulation of multi-agent systems has been proposed to provide a general framework for leader-following consensus in linear or nonlinear cases (Hong. Wang \& Jiang, 2013; Dong \& Huang, 2014). Internal model approach, developed to solve the conventional output regulation (Isidori, Marconi \& Serrani, 2003; Huang, 2004), was effectively used for the distributed design, especially for nonlinear agents. For example, a cooperative output regulation problem was considered for a class of nonlinear uncertain multi-agent systems with unity relative degree in Su \& Huang (2013), while agents in the output-feedback form were considered with an autonomous leader and no-loop graph (Ding, 2013).

\footnotetext{
‡ This work was supported in part by the Fundamental Research Funds for the Central Universities under Grant 24820152015RC36 and in part by National Natural Science Foundation of China under Grants 61503033, 61333001 and 61503359. This paper was not presented at any IFAC meeting. Tel.: +86-10-62651449. Fax.: +86-10-62587343.

Email addresses: yttang@bupt.edu.cn (Yutao Tang), yghong@iss.ac.cn (Yiguang Hong), xinghuw@ustc.edu.cn (Xinghu Wang).
}

It may be restrictive or unpractical if we always consider an autonomous leader without unknown inputs, especially in the case when the leader is an uncooperative target or contains unmodeled uncertainties. Therefore, it is necessary to study multi-agent control when the leader contains (unknown) inputs. In fact, the generalized output regulation (GOR) problem to track an exosystem (or a leader) with external inputs was discussed in many publications including Saberi, Stoorvogel \& Sannuti (2001) and Ramos, Celikovsky \& Kucera (2004). On the other hand, a distributed problem was investigated when the agent dynamics are double integrators to track a leader with an unknown but bounded acceleration in Cao \& Ren (2012), and then a similar design was given in Li, Liu, Ren \& Xie (2013) by assuming that the leader and followers share the same dynamics. To our best of knowledge, there are no general results on nonlinear multi-agent control when the unknown-input leader and the followers have different dynamics with external disturbances.

The objective of our paper is to study distributed output regulation for leader-following multi-agent systems with an unknown-input leader, whose dynamics is nonlinear and may differ from those of the followers. The contribution of the work is at least twofold:

- We extend the distributed output regulation to the case when the leader contains unknown inputs and has 
a dynamics different from those of the non-identical followers with (unbounded) local disturbances, and provide distributed controls to solve this problem in different cases. The results are consistent with the existing output regulation results when the leader does not have unknown inputs and the disturbances are bounded (e.g. Dong \& Huang, 2014; Su \& Huang, 2013).

- We extend the conventional GOR to its distributed version for multi-agent systems with an unknowninput leader. Moreover, both global and semi-global results are obtained for nonlinear agents with unity relative degree, while only local results were obtained for a conventional (single-agent) case in Ramos et al. (2004).

Notations: Let $\mathbb{R}^{n}$ be the $n$-dimensional Euclidian space and $\mathbb{R}_{M}^{n}=\left\{s \in \mathbb{R}^{n} \mid-M \leq s_{i} \leq M, i=1, \ldots, n\right\}$ for a constant $M>0$. For a vector $x,\|x\|\left(\right.$ or $\left.\|x\|_{\infty}\right)$ denotes its Euclidian norm (or infinite norm). $\operatorname{diag}\left\{b_{1}, \ldots, b_{n}\right\}$ denotes an $n \times n$ diagonal matrix with diagonal elements $b_{i}(i=1, \ldots, n) ; \operatorname{col}\left(a_{1}, \ldots, a_{n}\right)=\left[a_{1}^{T}, \ldots, a_{n}^{T}\right]^{T}$ for column vectors $a_{i}(i=1, \ldots, n)$. A continuous function $\alpha:[0, a) \rightarrow[0, \infty)$ belongs to class $\mathcal{K}$ if it is strictly increasing and $\alpha(0)=0$; It belongs to class $\mathcal{K}_{\infty}$ if it belongs to class $\mathcal{K}$ with $a=\infty$ and $\lim _{s \rightarrow \infty} \alpha(s) \rightarrow \infty$.

\section{Problem Formulation}

Consider a group of $n+1$ agents with one leader (regarded as node 0) as follows:

$$
\dot{v}=p(v)+q(v) w(t), \quad y_{0}=r(v, \mu)
$$

where $v \in \mathbb{R}^{n_{v}}$ is the leader's state, and $w(t) \in \mathbb{R}^{n_{w}}, y_{0} \in$ $\mathbb{R}$ are its input and output, respectively. Here $\mu \in \mathbb{R}^{n_{\mu}}$ is an uncertain parameter vector, and $w(t)$ is continuous satisfying $\|w(t)\|_{\infty} \leq l$ with a constant $l>0$. The other $n$ (non-identical) agents are followers described by

$$
\left\{\begin{array}{l}
\dot{z}_{i}=f_{i}\left(z_{i}, y_{i}, \mu\right) \\
\dot{y}_{i}=g_{i}\left(z_{i}, y_{i}, \mu\right)+b_{i} u_{i}+d_{i}, \quad i=1, \ldots, n
\end{array}\right.
$$

where $z_{i} \in \mathbb{R}^{n_{z_{i}}}, y_{i} \in \mathbb{R}, d_{i} \in \mathbb{R}, b_{i}>0$. Without loss of generality, we take $b_{i}=1$ and assume all functions $f_{i}$, $g_{i}, p, q, r$ are smooth with $f_{i}(0,0, \mu)=0, g_{i}(0,0, \mu)=0$, $p(0)=0, r(0, \mu)=0 . d_{i}$ is the local disturbance of agent $i$ governed by

$$
\dot{\omega}_{i}=S_{i} \omega_{i}, \quad d_{i}=D_{i}(\mu) \omega_{i}
$$

As usual, we assume $S_{i} \in \mathbb{R}^{n_{\omega_{i}} \times n_{\omega_{i}}}$ has no eigenvalues with negative real parts (Huang, 2004).

Clearly, the first-order nonlinear agent in Liu. Xie, Ren \& Wa $(2013)$ is a special case of (2), and system (2) was also considered to solve an output consensus problem for the exosystem without any inputs in Ding (2013).

The interaction topology among these agents can be described by a graph $\mathcal{G}=(\mathcal{V}, \mathcal{E})$, where $\mathcal{V}=\{0,1, \ldots, n\}$ is the set of nodes and $\mathcal{E}$ is the set of $\operatorname{arcs}$. $(i, j)$ denotes an arc leaving from node $i$ and entering node $j$ (Godsil \& Rovle, 2001). A walk in graph $\mathcal{G}$ is an alternating sequence $i_{1} e_{1} i_{2} e_{2} \cdots e_{k-1} i_{k}$ of nodes $i_{l}$ and arcs $e_{m}=$ $\left(i_{m}, i_{m+1}\right) \in \mathcal{E}$ for $l=1,2, \ldots, k$. If there exists a walk from node $i$ to node $j$ then node $i$ is said to be reachable from $j$. Define the neighbor set of agent $i$ as $\mathcal{N}_{i}=\{j:$ $(j, i) \in \mathcal{E}\}$ for $i=1, \ldots, n$. A weighted adjacency matrix of $\mathcal{G}$ is denoted by $A=\left[a_{i j}\right] \in \mathbb{R}^{(n+1) \times(n+1)}$, where $a_{i i}=0$ and $a_{i j} \geq 0\left(a_{i j}>0\right.$ if $\left.(j, i) \in \mathcal{E}\right)$. A graph is said to be undirected if $a_{i j}=a_{j i}(i, j=0,1, \ldots, n)$. The Laplacian $L=\left[l_{i j}\right] \in \mathbb{R}^{(n+1) \times(n+1)}$ of graph $\mathcal{G}$ is defined as $l_{i i}=\sum_{j \neq i} a_{i j}$ and $l_{i j}=-a_{i j}(j \neq i)$. Denote $\overline{\mathcal{G}}$ as the induced subgraph of $\mathcal{G}$ associated with the $n$ followers. The following assumption has been widely used in coordination of multi-agent systems Hong, Hu \& Gao, 2006; Su \& Huang, 2013).

Assumption 1 The leader (node 0) is reachable from any other node of $\mathcal{G}$ and the induced subgraph $\overline{\mathcal{G}}$ is undirected.

Given a communication graph $\mathcal{G}$, denote $H \in \mathbb{R}^{n \times n}$ as the submatrix of the Laplacian $L$ by deleting its first row and first column. By Lemma 3 in Hong et al. (2006), $H$ is positive definite under Assumption 1. The distributed control law can be constructed as follows:

$$
\begin{gathered}
u_{i}=k_{i}\left(\xi_{i}, y_{i}-y_{j}, j \in \mathcal{N}_{i}\right), \\
\dot{\xi}_{i}=h_{i}\left(\xi_{i}, y_{i}-y_{j}, j \in \mathcal{N}_{i}\right)
\end{gathered}
$$

where $\xi_{i} \in \mathbb{R}^{n_{\xi_{i}}}$ with a nonnegative integer $n_{\xi_{i}}$ and functions $k_{i}(\cdot), h_{i}(\cdot)$ to be designed later.

To handle this nonlinear multi-agent system with an unknown-input leader, we formulate the problem as the distributed generalized output regulation problem or simply distributed regulation problem. It is said to be (globally) solved for systems (1)-(3) with a given graph $\mathcal{G}$, if we can find a distributed control law (4), such that, for any $\left(z_{i}(0), y_{i}(0)\right) \in \mathbb{R}^{n_{z_{i}}+1}, \mu \in \mathbb{R}^{n_{\mu}}, \xi_{i}(0) \in \mathbb{R}^{n_{\xi_{i}}}$, $v(0) \in \mathbb{R}^{n_{v}}, \omega_{i}(0) \in \mathbb{R}^{n_{\omega_{i}}}$, the trajectory of the closedloop system, composed of (1), (2), and (4), is well-defined for all $t>0$, and moreover,

$$
\lim _{t \rightarrow \infty} e_{i}(t)=0, \quad e_{i}=y_{i}-y_{0}, \quad i=1, \ldots, n
$$

Our problem is said to be semi-globally solved if, for any given $M>0$, we can find a control law (4) with a constant $\bar{M} \geq 0$, such that, for any initial condition $\left(z_{i}(0), y_{i}(0)\right) \in \mathbb{R}_{M}^{n_{z_{i}}+1}, \mu \in \mathbb{R}_{M}^{n_{\mu}}, \xi_{i}(0) \in \mathbb{R}_{\bar{M}}^{n_{\xi_{i}}}, v(0) \in$ $\operatorname{Var}_{n}^{n} v$ and $\omega_{i}(0) \in \mathbb{R}_{M}^{n_{\omega_{i}}}$, the trajectory of the closed-loop system is well-defined for all $t>0$ and (5) holds. 
Remark 1 When $n=1$, our problem becomes $G O R$ studied in Saberi et al. (2001) and Ramos et al. (2004). Here we seek non-local output feedback control for nonlinear systems of the form (2), while only local results were obtained in Ramos et al. (2004) requiring the exosystem's state.

Remark 2 Because of the unknown expression or type of $w(t)$, adaptive IM discussed in Su ES Huang (2013) fails to solve our problem even with a time-varying one like in Yang ES Huand (2010). In fact, our problem can be viewed as a distributed version of GOR to handle those exosystems (or leaders) with unknown inputs, which certainly extends the existing multi-agent output regulation formulation when the leaders have no unknown inputs (Honq et al., 2013; Su \& Huand, 2013).

The following assumption was used for GOR of nonlinear systems (see Ramos et al., 2004).

Assumption 2 There exist two class $\mathcal{K}$ functions $\alpha_{0}(\cdot)$ and $\gamma_{0}(\cdot)$ such that

$$
\|v(t)\| \leq \alpha_{0}(\|v(0)\|)+\gamma_{0}\left(\|w(t)\|_{\infty}\right) .
$$

Clearly, $v(t)$ is bounded by $\bar{b}_{1}=\alpha_{0}(\|v(0)\|)+\gamma_{0}(l)$ and $\bar{b}_{2}=\max _{v \in \mathbb{R}_{\bar{b}_{1}}^{n_{v}}}\left\{\frac{\partial r}{\partial v} q(v)\right\}$ is well-defined under this assumption. Moreover, system (1) is Lyapunov stable at $v=0$ when $w=0$ with the neutrally stable exosystem for nonlinear output regulation as one of its special cases.

Similar to the output regulation problem, the solvability of regulator equations plays a key role in the study of nonlinear GOR. Therefore, we give the following assumption for the solution of regulator equations.

Assumption 3 For $i=1, \ldots, n$, there exists a smooth function $\mathbf{z}_{i}(v, \mu)$ with $\mathbf{z}_{i}(0, \mu)=0$ such that,

$$
\frac{\partial \mathbf{z}_{i}(v, \mu)}{\partial v} p(v)=f_{i}\left(\mathbf{z}_{i}(v, \mu), r(v, \mu)\right)
$$

Under Assumption 3, letting $\mathbf{u}_{i}(v, \mu)=\frac{\partial r(v, \mu)}{\partial v} p(v)-$ $g_{i}\left(\mathbf{z}_{i}(v, \mu), r(v, \mu)\right)$ and performing a coordinate transformation: $\bar{z}_{i}=z_{i}-\mathbf{z}_{i}(v, \mu), e_{i}=y_{i}-r(v, \mu)$ gives

$$
\left\{\begin{array}{l}
\dot{\bar{z}}_{i}=\bar{f}_{i}\left(\bar{z}_{i}, e_{i}, v, w, \mu\right) \\
\dot{e}_{i}=\bar{g}_{i}\left(\bar{z}_{i}, e_{i}, v, w, \mu\right)+u_{i}+d_{i}
\end{array}\right.
$$

where

$$
\begin{aligned}
\bar{f}_{i}\left(\bar{z}_{i}, e_{i}, v, w, \mu\right) & =\hat{f}_{i}\left(\bar{z}_{i}, e_{i}, v, \mu\right)-\frac{\partial \mathbf{z}_{i}(v, \mu)}{\partial v} q(v) w \\
\bar{g}_{i}\left(\bar{z}_{i}, e_{i}, v, w, \mu\right) & =\hat{g}_{i}\left(\bar{z}_{i}, e_{i}, v, \mu\right)-\mathbf{u}_{i}(v, \mu)-\frac{\partial r(v, \mu)}{\partial v} q(v) w \\
\hat{f}_{i}\left(\bar{z}_{i}, e_{i}, v, \mu\right) & =f_{i}\left(z_{i}, y_{i}, \mu\right)-f_{i}\left(\mathbf{z}_{i}(v, \mu), r(v, \mu), \mu\right) \\
\hat{g}_{i}\left(\bar{z}_{i}, e_{i}, v, \mu\right) & =g_{i}\left(z_{i}, y_{i}, \mu\right)-g_{i}\left(\mathbf{z}_{i}(v, \mu), r(v, \mu), \mu\right) .
\end{aligned}
$$

Furthermore, $\bar{f}_{i}(0,0, v, 0, \mu)=0, \bar{g}_{i}(0,0, v, 0, \mu)=0$, $\hat{g}_{i}(0,0, v, \mu)=0$. For simplicity, the error system can be rewritten as

$$
\left\{\begin{array}{l}
\dot{\bar{z}}=\bar{f}(\bar{z}, e, v, w, \mu) \\
\dot{e}=\bar{g}(\bar{z}, e, v, w, \mu)+u+d
\end{array}\right.
$$

where $\bar{z}=\operatorname{col}\left(\bar{z}_{1}, \ldots, \bar{z}_{n}\right), e=\operatorname{col}\left(e_{1}, \ldots, e_{n}\right), u=$ $\operatorname{col}\left(u_{1}, \ldots, u_{n}\right), d=\operatorname{col}\left(d_{1}, \ldots, d_{n}\right)$ and $\bar{f}, \bar{g}$ are suitably defined by (7).

By (8), output regulation problem of the multi-agent system (1), (2) and (3) is transformed into a problem of finding a distributed control law in the form of (4) such that $\lim _{t \rightarrow \infty} e(t)=0$ and the trajectory of the closedloop system is well-defined for $t>0$. For this purpose, we introduce the following assumption for the zero dynamics (i.e., the $\bar{z}$-subsystem) of system (8), though we need not "stabilize" this subsystem (to make $\bar{z}$ vanish).

Assumption 4 For any compact subset $\Sigma \subset \mathbb{R}^{\hat{n}}$ $\left(\hat{n}=n_{v}+n_{w}+n_{\mu}\right)$, and for $i=1, \ldots, n$, there exists a smooth Lyapunov function $V_{\bar{z}_{i}}(\cdot)$ satisfying $\alpha_{1 i}\left(\left\|\bar{z}_{i}\right\|\right) \leq V_{\bar{z}_{i}}\left(\bar{z}_{i}\right) \leq \alpha_{2 i}\left(\left\|\bar{z}_{i}\right\|\right)$ for some smooth functions $\alpha_{1 i}(\cdot), \alpha_{2 i}(\cdot) \in \mathcal{K}_{\infty}$, such that, for any $(v(t), w(t), \mu) \in \Sigma$

$$
\left.\dot{V}_{\bar{z}_{i}}\left(\bar{z}_{i}\right)\right|_{(7)} \leq-\alpha_{i}\left(\left\|\bar{z}_{i}\right\|\right)+\delta_{1 i} \gamma_{1 i}\left(e_{i}\right)+\delta_{2 i} \gamma_{2 i}(w)
$$

where $\gamma_{1 i}(\cdot), \gamma_{2 i}(\cdot)$ are known smooth positive definite functions, $\alpha_{i}(\cdot)$ is a known class $\mathcal{K}_{\infty}$ function satisfying $\limsup _{s \rightarrow 0+}\left(\alpha_{i}^{-1}\left(s^{2}\right) / s\right) \leq \infty$, and $\delta_{1 i}, \delta_{2 i}$ are some unknown positive constants.

Remark 3 Although the $\bar{z}_{i}$-subsystem is related with $v$, the condition (9) is not restrictive since $v(t)$ is bounded by Assumption 2 and $\bar{f}_{i}(0,0, v, 0, \mu)=0$. Similar assumptions were commonly used in the study of nonlinear output regulation (e.g. Donq \&5 Huand, 2014; Su \& Huand, 2013; Xu \&3 Huand, 2010).

\section{Main Results}

In this section, we give a constructive design to solve our problem by investigating system (8).

For the following non-smooth analysis, consider an equation $\dot{x}=f(x, t)$ with a discontinuous righthand side, where $f: \mathbb{R}^{m} \times \mathbb{R} \rightarrow \mathbb{R}^{m}$ is measurable and essentially locally bounded. By Proposition 3 in Cortes (2008), it has a Filippov solution on $\left[t_{0}, t_{1}\right]$. Let $V: \mathbb{R}^{m} \rightarrow \mathbb{R}$ be a locally Lipschitz continuous function. $\dot{V} \triangleq \bigcap_{\xi \in \partial V} \xi^{T} \mathcal{F}[f](z, t)$ represents the set-valued Lie derivative of $V$, where $\partial V(z)$ denotes the Clarke's generalized gradient of $V$ (Cortes, 2008).

Then we start with a general discussion and give a simpler design for a special case. 


\subsection{General Discussion}

Here each agent only knows that the unknown leader's input is bounded, but does not know the exact value of the input bound.

To track the leader and meanwhile reject the (unbounded) local disturbance $d_{i}$, we split the total control effort into two parts as $u_{i}=u_{i}^{d}+u_{i}^{r}$, where the term $u_{i}^{d}$ is to deal with $d_{i}$ and $u_{i}^{r}$ to make $y_{i}$ follow $y_{0}$. It is well-known that internal model methods were effective to reject modeled disturbances. Here we construct $u_{i}^{d}$ following the same technical line.

Let $P_{i}(s)=s^{n_{p_{i}}}+\hat{p}_{i, 1} s^{n_{p_{i}}-1}+\cdots+\hat{p}_{i, n_{p_{i}}-1} s+\hat{p}_{i, n_{p_{i}}}$ be the minimal polynomial of the matrix $S_{i}$ and denote $\tau_{i}=\operatorname{col}\left(\tau_{i, 1}, \ldots, \tau_{i, n_{p_{i}}}\right)$ with $\tau_{i, j}=\frac{\mathrm{d}^{j-1} d_{i}(t)}{\mathrm{d} t^{j-1}}$. Take

$$
\Phi_{i}=\left[\begin{array}{c|c}
0 & I_{n_{p_{i}}-1} \\
\hline-\hat{p}_{i, n_{p_{i}}} & \hat{\mathbf{p}}_{i}
\end{array}\right], \Psi_{i}=\left[\begin{array}{ll}
1 & 0_{1 \times\left(n_{p_{i}}-1\right)}
\end{array}\right]
$$

where $\hat{\mathbf{p}}_{i}=\left[-\hat{p}_{i, n_{p_{i}}-1} \cdots-\hat{p}_{i, 1}\right]$. By a direct calculation, we obtain

$$
\dot{\tau}_{i}=\Phi_{i} \tau_{i}, \quad d_{i}=\Psi_{i} \tau_{i}
$$

The system (10) is called a steady-state generator in Huang (2004), which helps us reject the unwanted disturbance $d_{i}$. Since the pair $\left(\Psi_{i}, \Phi_{i}\right)$ is observable, there exists a constant matrix $G_{i}$ such that $F_{i}=\Phi_{i}+G_{i} \Psi_{i}$ is Hurwitz. To asymptotically reject the disturbance $d_{i}$, let

$$
u_{i}^{d}=-\Psi_{i} \eta_{i}, \quad \dot{\eta}_{i}=F_{i} \eta_{i}+G_{i} u_{i}
$$

Inspired by the robust adaptive control law used in Jiang \& Hill (1999), we propose the tracking control with a constant $\lambda>0$ for agent $i(i=1, \ldots, n)$ :

$$
\begin{aligned}
& u_{i}^{r}=-k_{i} \rho_{i}\left(e_{v i}\right) e_{v i}-\theta_{i} \operatorname{sgn}\left(e_{v i}\right) \\
& \dot{k}_{i}=-\lambda k_{i}+\rho_{i}\left(e_{v i}\right) e_{v i}^{2}, \quad \dot{\theta}_{i}=\left|e_{v i}\right|
\end{aligned}
$$

where $e_{v i}=\sum_{j \in \mathcal{V}} a_{i j}\left(y_{i}-y_{j}\right)$. For simplicity, we take $k_{i}(0)=\theta_{i}(0)=0$ (see Praly, 2003 for a similar setting).

Then the control law for agent $i$ can be written as

$$
\begin{aligned}
& u_{i}=-\Psi_{i} \eta_{i}-k_{i} \rho_{i}\left(e_{v i}\right) e_{v i}-\theta_{i} \operatorname{sgn}\left(e_{v i}\right) \\
& \dot{\eta}_{i}=F_{i} \eta_{i}+G_{i} u_{i} \\
& \dot{k}_{i}=-\lambda k_{i}+\rho_{i}\left(e_{v i}\right) e_{v i}^{2}, \quad \dot{\theta}_{i}=\left|e_{v i}\right| .
\end{aligned}
$$

Remark 4 Different from most existing internal model (IM) design for multi-agent output regulation (e.g. Dona \& Huand, 2014; Su \& Huand, 2013), the control (13) contains two parts: the IM design $u_{i}^{d}$ for disturbance rejection and the non-smooth design $u_{i}^{r}$ to handle the leader's unknown inputs. The gains $k_{i}$ and $\theta_{i}$ are designed and updated, independent of $\omega_{i}(0), v(0)$, and $l$ (refer Xu \& Huand, 2010 and Tanq, 2014 for similar techniques).

To select a proper positive function $\rho_{i}(\cdot)$, we perform a coordinate transformation $\hat{\eta}_{i}=\eta_{i}-\tau_{i}-G_{i} e_{i}$ and the composite system of agent $i$ becomes

$$
\left\{\begin{array}{l}
\dot{\bar{z}}_{i}=\bar{f}_{i}\left(\bar{z}_{i}, e_{i}, v, w, \mu\right) \\
\dot{\hat{\eta}}_{i}=F_{i} \hat{\eta}_{i}+F_{i} G_{i} e_{i}-G_{i} \bar{g}_{i}\left(\bar{z}_{i}, e_{i}, v, w, \mu\right) \\
\dot{e}_{i}=\bar{g}_{i}\left(\bar{z}_{i}, e_{i}, v, w, \mu\right)-\Psi_{i} \hat{\eta}_{i}-\Psi_{i} G_{i} e_{i}+u_{i}^{r} \\
\dot{k}_{i}=-\lambda k_{i}+\rho_{i}\left(e_{v i}\right) e_{v i}^{2}, \quad \dot{\theta}_{i}=\left|e_{v i}\right|
\end{array}\right.
$$

Taking $\hat{z}_{i}=\operatorname{col}\left(\bar{z}_{i}, \hat{\eta}_{i}\right)$, we have the following result.

Lemma 1 Under Assumption 4, for any compact subset $\Sigma \subset \mathbb{R}^{\hat{n}}$, there are smooth Lyapunov functions $V_{\hat{z}_{i}}(\cdot)$ satisfying $\hat{\alpha}_{1 i}\left(\left\|\hat{z}_{i}\right\|\right) \leq V_{\hat{z}_{i}}\left(\hat{z}_{i}\right) \leq \hat{\alpha}_{2 i}\left(\left\|\hat{z}_{i}\right\|\right)$ for some smooth functions $\hat{\alpha}_{1 i}(\cdot)$ and $\hat{\alpha}_{2 i}(\cdot) \in \mathcal{K}_{\infty}$ with $i=1, \ldots, n$ such that, for any $(v(t), w(t), \mu) \in \Sigma$ and $\omega_{i}(t) \in \mathbb{R}^{n_{\omega_{i}}}$,

$$
\left.\dot{V}_{\hat{z}_{i}}\left(\hat{z}_{i}\right)\right|_{(14)} \leq-\left\|\hat{z}_{i}\right\|^{2}+\hat{\delta}_{1 i} \hat{\gamma}_{1 i}\left(e_{i}\right)+\hat{\delta}_{2 i} \hat{\gamma}_{2 i}(w)+\hat{\delta}_{3 i}
$$

where $\hat{\gamma}_{1 i}(\cdot)$ and $\hat{\gamma}_{2 i}(\cdot)$ are known smooth positive definite functions, and $\hat{\delta}_{1 i}, \hat{\delta}_{2 i}, \hat{\delta}_{3 i}$ are unknown positive constants.

Proof. From $(7)$ and $\hat{g}_{i}(0,0, v, \mu)=0$, by Lemma 7.8 in Huang (2004), there are known positive smooth functions $\hat{\phi}_{1 i}(\cdot), \hat{\phi}_{2 i}(\cdot)$ and an unknown constant $\hat{c}_{i}>0$ such that

$$
\begin{aligned}
& \left\|F_{i} G_{i} e_{i}-G_{i} \bar{g}_{i}\left(\bar{z}_{i}, e_{i}, v, w, \mu\right)\right\| \\
& \leq \hat{c}_{i}\left(\hat{\phi}_{1 i}\left(\bar{z}_{i}\right)\left\|\bar{z}_{i}\right\|+\hat{\phi}_{2 i}\left(e_{i}\right)\left|e_{i}\right|+\|w\|+1\right) .
\end{aligned}
$$

Based on Assumption 4 and by the changing supply functions technique (Sontag \& Teel, 1995), for any smooth function $\Delta_{i}\left(\bar{z}_{i}\right)>0$, there exists a smooth function $W_{\bar{z}_{i}}\left(\bar{z}_{i}\right)$ satisfying $\bar{\alpha}_{3 i}\left(\left\|\bar{z}_{i}\right\|\right) \leq W_{\bar{z}_{i}}\left(\bar{z}_{i}\right) \leq \bar{\alpha}_{4 i}\left(\left\|\bar{z}_{i}\right\|\right)$ for some smooth functions $\bar{\alpha}_{3 i}(\cdot), \bar{\alpha}_{4 i}(\cdot) \in \mathcal{K}_{\infty}$ such that

$$
\begin{aligned}
\left.\dot{W}_{\bar{z}_{i}}\left(\bar{z}_{i}\right)\right|_{(7) \leq} & -\Delta_{i}\left(\bar{z}_{i}\right)\left\|\bar{z}_{i}\right\|^{2} \\
& +\bar{\delta}_{3 i} \bar{\gamma}_{3 i}\left(e_{i}\right) e_{i}{ }^{2}+\bar{\delta}_{4 i} \bar{\gamma}_{4 i}(w)\|w\|^{2}
\end{aligned}
$$

where $\bar{\delta}_{3 i}, \bar{\delta}_{4 i}$ are some unknown positive constants, and $\bar{\gamma}_{3 i}(\cdot), \bar{\gamma}_{4 i}(\cdot)$ are known smooth positive functions.

Let $V_{\hat{z}_{i}}\left(\hat{z}_{i}\right)=\kappa_{i} W_{\bar{z}_{o}}\left(\bar{z}_{i}\right)+2 \hat{\eta}_{i}^{T} P_{i} \hat{\eta}_{i}$ with a constant $\kappa_{i}>$ 0 to be determined, where $P_{i}$ is the positive definite solution of the Lyapunov equation $P_{i} F_{i}+F_{i}^{T} P_{i}=-I_{n_{p_{i}}}$. Clearly, there exist smooth functions $\hat{\alpha}_{1 i}(\cdot), \hat{\alpha}_{2 i}(\cdot) \in \mathcal{K}_{\infty}$ satisfying $\hat{\alpha}_{1 i}\left(\left\|\hat{z}_{i}\right\|\right) \leq V_{\hat{z}_{i}}\left(\hat{z}_{i}\right) \leq \hat{\alpha}_{2 i}\left(\left\|\hat{z}_{i}\right\|\right)$. Using (15)- 
(16) for all $(v(t), w(t), \mu) \in \Sigma, \omega_{i}(t) \in \mathbb{R}^{n_{\omega_{i}}}$, we have

$$
\begin{aligned}
\left.\dot{V}_{\hat{z}_{i}}\left(\hat{z}_{i}\right)\right|_{(14)} & \leq-\left[\kappa_{i} \Delta_{i}\left(\bar{z}_{i}\right)-4 \hat{c}_{i}^{2}\left\|P_{i}\right\|^{2} \hat{\phi}_{1 i}^{2}\left(\bar{z}_{i}\right)\right]\left\|\bar{z}_{i}\right\|^{2} \\
& -\left\|\hat{\eta}_{i}\right\|^{2}+\left[\kappa_{i} \bar{\delta}_{3 i} \bar{\gamma}_{3 i}\left(e_{i}\right)+4 \hat{c}_{i}^{2}\left\|P_{i}\right\|^{2} \hat{\phi}_{2 i}^{2}\left(e_{i}\right)\right] e_{i}{ }^{2} \\
& +\left[\kappa_{i} \bar{\delta}_{4 i} \bar{\gamma}_{4 i}(w)+4 \hat{c}_{i}^{2}\left\|P_{i}\right\|^{2}\right]\|w\|^{2}+4 \hat{c}_{i}^{2}\left\|P_{i}\right\|^{2}
\end{aligned}
$$

Letting $\kappa_{i} \geq \max \left\{1,4 \hat{c}_{i}^{2}\left\|P_{i}\right\|^{2}\right\}, \Delta_{i}\left(\bar{z}_{i}\right) \geq 1+\hat{\phi}_{1 i}^{2}\left(\bar{z}_{i}\right)$, $\hat{\delta}_{1 i} \geq \max \left\{\kappa_{i} \bar{\delta}_{3 i}, 4 \hat{c}_{i}^{2}\left\|P_{i}\right\|^{2}\right\}, \hat{\delta}_{2 i} \geq \max \left\{\kappa_{i} \bar{\delta}_{4 i}, 4 \hat{c}_{i}^{2}\left\|P_{i}\right\|^{2}\right\}$, $\hat{\delta}_{3 i} \geq 4 \hat{c}_{i}^{2}\left\|P_{i}\right\|^{2}$ and $\hat{\gamma}_{1 i} \geq\left[\bar{\gamma}_{3 i}\left(e_{i}\right)+\hat{\phi}_{2 i}^{2}\left(e_{i}\right)\right]\left|e_{i}\right|^{2}$, $\hat{\gamma}_{2 i} \geq\left[\bar{\gamma}_{4 i}(w)+1\right]\|w\|^{2}$ yields the conclusion.

It is not hard to check that there is a Filippov solution of the closed-loop system consisting of (1), (2), and (13). Then we present one of our main results.

Theorem 1 Under Assumptions 1-4, there exist smooth positive functions $\rho_{i}(\cdot)$ for $i=1, \ldots, n$ such that our problem is globally solved by the distributed control (13).

Proof. The proof will be given in two steps.

Step 1: We first constructively give smooth positive functions $\rho_{i}(\cdot)$ for $i=1, \ldots, n$ and show the existence of the trajectory $\left(z_{i}, y_{i}, k_{i}, \eta_{i}\right)$ for $t>0$. Since $\eta_{i}$-subsystem is linear with a Hurwitz system matrix, we only have to show the boundedness of $\left(z_{i}, y_{i}, k_{i}\right)$. For this purpose, we seek to prove the boundedness of $(\bar{z}, e, \bar{k})$, where $\bar{k}=\operatorname{col}\left(\bar{k}_{1}, \ldots, \bar{k}_{n}\right), \bar{k}_{i}=k_{i}-K$ and $K>0$ is a constant to be determined.

From Assumption 2, we can always find a compact subset $\Sigma \subset \mathbb{R}^{\hat{n}}$ containing $(v(t), w(t), \mu)$ for $t>0$. Based on Assumption 4, Lemma 1 and the changing supply functions technique (Sontag \& Teel, 1995), for any smooth function $\Delta_{i}\left(\hat{z}_{i}\right)>0$, there is a smooth function $W_{\hat{z}_{i}}\left(\hat{z}_{i}\right)$ satisfying $\hat{\alpha}_{3 i}\left(\left\|\hat{z}_{i}\right\|\right) \leq W_{\hat{z}_{i}}\left(\hat{z}_{i}\right) \leq \hat{\alpha}_{4 i}\left(\left\|\hat{z}_{i}\right\|\right)$ for some smooth functions $\hat{\alpha}_{3 i}(\cdot)$ and $\hat{\alpha}_{4 i}(\cdot) \in \mathcal{K}_{\infty}$ such that

$$
\begin{aligned}
\left.\dot{W}_{\hat{z}_{i}}\left(\hat{z}_{i}\right)\right|_{(14)} & \leq-\Delta_{i}\left(\hat{z}_{i}\right)\left\|\hat{z}_{i}\right\|^{2}+\hat{\delta}_{4 i} \hat{\gamma}_{3 i}\left(e_{i}\right) e_{i}{ }^{2} \\
& +\hat{\delta}_{5 i} \hat{\gamma}_{4 i}(w)\|w\|^{2}+\hat{\delta}_{6 i}
\end{aligned}
$$

where $\hat{\delta}_{4 i}, \hat{\delta}_{5 i}, \hat{\delta}_{6 i}$ are some unknown positive constants, and $\hat{\gamma}_{3 i}(\cdot), \hat{\gamma}_{4 i}(\cdot)$ are known smooth positive functions.

Let $W_{0}(\hat{z})=\sum_{i=1}^{n} W_{\hat{z}_{i}}\left(\hat{z}_{i}\right), V_{0}(e)=e^{T} H e$. By Young's inequality, the set-valued Lie derivative of $V_{0}$ satisfies

$$
\left.\dot{V}_{0}\right|_{(14)} \leq 3 \sum_{i=1}^{n} e_{v i}^{2}+\sum_{i=1}^{n}\left\|\Upsilon_{1 i}\right\|^{2}+\Xi_{1}+\bigcap_{\xi \in \partial V_{0}} \xi^{T} u^{r}
$$

where $\Upsilon_{1 i} \triangleq \hat{g}_{i}\left(\bar{z}_{i}, e_{i}, v, \mu\right)-\Psi_{i} \hat{\eta}_{i}-\Psi_{i} G_{i} e_{i}$ and $\Xi_{1} \triangleq$ $\sum_{i=1}^{n}\left(\bar{b}_{2}^{2}\|w\|^{2}+\left\|\mathbf{u}_{i}(v, \mu)\right\|^{2}\right)$. Since $\hat{g}_{i}(0,0, v, \mu)=0$ and $v$ is bounded, by Lemma $7.8 \mathrm{in}$ Huang (2004), there exist known positive smooth functions $\phi_{1 i}(\cdot), \phi_{2 i}(\cdot)$ with an unknown positive constant $c_{i}$ such that

$$
\left\|\Upsilon_{1 i}\right\|^{2} \leq c_{i}\left(\phi_{1 i}\left(\hat{z}_{i}\right)\left\|\hat{z}_{i}\right\|^{2}+\phi_{2 i}\left(e_{i}\right)\left\|e_{i}\right\|^{2}\right) .
$$

Note that

$$
\sum_{i=1}^{n}\left[\hat{\delta}_{4 i} \hat{\gamma}_{3 i}\left(e_{i}\right)+c_{i} \phi_{2 i}\left(e_{i}\right)\right] e_{i}{ }^{2} \leq \sum_{i=1}^{n} \delta_{i} \hat{\phi}_{i}\left(e_{i}\right) e_{i}{ }^{2}
$$

with $\delta_{i}=\hat{\delta}_{4 i}+c_{i}$ and a positive smooth function $\hat{\phi}_{i}\left(e_{i}\right) \geq$ $\max \left\{\hat{\gamma}_{3 i}\left(e_{i}\right), \phi_{2 i}\left(e_{i}\right)\right\}$. Recalling $e_{v}=H e$, by similar arguments, we obtain

$$
\sum_{i=1}^{n}\left[\hat{\delta}_{4 i} \hat{\gamma}_{3 i}\left(e_{i}\right)+c_{i} \phi_{2 i}\left(e_{i}\right)\right] e_{i}{ }^{2} \leq \sum_{i=1}^{n} \delta_{i} \tilde{\phi}_{i}\left(e_{v i}\right) e_{v i}^{2}
$$

for some known positive smooth functions $\tilde{\phi}_{i}(\cdot)$.

Construct a positive definite and radially unbounded Lyapunov function candidate as $V(\hat{z}, e, \bar{k})=W_{0}(\hat{z})+$ $V_{0}(e)+\sum_{i=1}^{n} \bar{k}_{i}^{2}$. Clearly, $\theta_{i} \geq 0$, and by (17)-(19), it follows

$$
\begin{aligned}
\left.\dot{V}\right|_{(14)} \leq & \sum_{i=1}^{n}-\left[\Delta_{i}\left(\bar{z}_{i}\right)-c_{i} \phi_{1 i}\left(\hat{z}_{i}\right)\right]\left\|\hat{z}_{i}\right\|^{2}-\sum_{i=1}^{n} \lambda \bar{k}_{i}^{2} \\
& -\sum_{i=1}^{n}\left[2 K \rho\left(e_{v i}\right)-3-\delta_{i} \tilde{\phi}_{i}\left(e_{v i}\right)\right] e_{v i}^{2}+\Xi_{2}
\end{aligned}
$$

where

$$
\Xi_{2} \triangleq \Xi_{1}+\sum_{i=1}^{n} \hat{\delta}_{5 i} \hat{\gamma}_{4 i}(w)\|w\|^{2}+\sum_{i=1}^{n} \hat{\delta}_{6 i}+\sum_{i=1}^{n} \lambda K^{2} .
$$

For $i=1, \ldots, n$, we take $\Delta_{i}, \rho_{i}$ and $K$ with $\Delta_{i}\left(\hat{z}_{i}\right)=$ $-c_{i} \phi_{1 i}\left(\hat{z}_{i}\right)+1, \rho_{i}\left(e_{v i}\right)=\tilde{\phi}_{i}\left(e_{v i}\right)+1, K>\frac{1}{2}\left(\delta_{i}+4\right)$. Then

$$
\left.\dot{V}\right|_{(14)} \leq-\sum_{i=1}^{n}\left\|\hat{z}_{i}\right\|^{2}-\sum_{i=1}^{n} e_{v i}^{2}-\sum_{i=1}^{n} \lambda \bar{k}_{i}^{2}+\Xi_{2} .
$$

Similar to the analysis of Theorem 4.14 in Khalil (2002), for any $\lambda>0$, the uniform boundedness of $\hat{z}, e$ and $\vec{k}$ with any solution of the closed-loop system is obtained, which implies the boundedness of $z_{i}, y_{i}$, and $k_{i}$.

Step 2: Let us check $e$ under the controller (13). Take a positive definite function $V_{1}(e, \bar{\theta})=V_{0}(e)+\sum_{i=1}^{n} \bar{\theta}_{i}^{2}$, where $\bar{\theta}_{i}=\theta_{i}-\Theta$ with $\Theta>0$ to be specified later. Then

$$
\begin{aligned}
\left.\dot{V}_{1}\right|_{(14)} & =2 \sum_{i=1}^{n} e_{v i}\left[\Upsilon_{2 i}-\theta_{i} \operatorname{sgn}\left(e_{v i}\right)\right]+2 \sum_{i=1}^{n} \bar{\theta}_{i} \dot{\theta}_{i} \\
& \leq 2 \sum_{i=1}^{n} e_{v i}\left[\Upsilon_{2 i}-\Theta \operatorname{sgn}\left(e_{v i}\right)\right]
\end{aligned}
$$


where $\Upsilon_{2 i} \triangleq \bar{g}_{i}\left(\bar{z}_{i}, e_{i}, v, w, \mu\right)-\Psi_{i} \hat{\eta}_{i}-\Psi_{i} G_{i} e_{i}-$ $k_{i} \rho_{i}\left(e_{v i}\right) e_{v i}$.

From the boundedness of $\hat{z}, e, v, \omega, \mu$ and the continuity of related functions, there is a positive constant $\hat{M}$ such that $\left\|\Upsilon_{2 i}\right\| \leq \hat{M}$ for all $i$. Letting $\Theta>\hat{M}+1$ gives

$$
\left.\dot{V}_{1}\right|_{(14)} \leq-2 \sum_{i=1}^{n}(\Theta-\hat{M})\left|e_{v i}\right| \leq-2 \sum_{i=1}^{n}\left|e_{v i}\right| .
$$

Because $V_{1}$ is radially unbounded, $\theta_{i}$ is bounded. From the boundedness of $e$ showed above, $\dot{e}_{i}$ is also bounded by (13) and (14), and then we can obtain the uniform continuity of $V_{2} \triangleq 2 \sum_{i=1}^{n}\left|e_{v i}\right|$ with respect to time $t$. Integrating (20) from 0 to $t$ and taking $t \rightarrow \infty$, we have

$$
\int_{0}^{\infty} V_{2}(e(t)) \mathrm{d} t \leq V_{1}(0) .
$$

Recalling the Barbalat's lemma (Khalil, 2002), $V_{2}(e(t)) \rightarrow$ 0 when $t \rightarrow \infty$, and hence $e_{i}$ converges to zero as $t \rightarrow \infty$ for $i=1, \ldots, n$. Thus, the conclusion is obtained.

\subsection{Special Case}

In some cases, we may know the domain of the initial condition $\left(z_{i}(0), y_{i}(0)\right), v(0), \omega_{i}(0)$ and the bound of the unknown input and the uncertain parameter $\mu$. Of course, we can still use the proposed control law (13), but here we construct a simpler control law to solve the problem based on the additional information. To this end, it is reasonable to introduce a new assumption to replace Assumption 4.

Assumption 5 Given any compact subset $\Sigma \subset \mathbb{R}^{\hat{n}}$, there exist smooth Lyapunov functions $V_{\bar{z}_{i}}(\cdot)$ satisfying $\alpha_{1 i}\left(\left\|\bar{z}_{i}\right\|\right) \leq V_{\bar{z}_{i}}\left(\bar{z}_{i}\right) \leq \alpha_{2 i}\left(\left\|\bar{z}_{i}\right\|\right)$ for some smooth functions $\alpha_{1 i}(\cdot)$ and $\alpha_{2 i}(\cdot) \in \mathcal{K}_{\infty}($ for $i=1, \ldots, n)$ such that, for any $(v(t), w(t), \mu) \in \Sigma$,

$$
\left.\dot{V}_{\bar{z}_{i}}\right|_{(7)} \leq-\alpha_{i}\left(|| \bar{z}_{i}||\right)+\gamma_{1 i}\left(e_{i}\right)+\gamma_{2 i}(w)
$$

where $\gamma_{1 i}(\cdot)$ and $\gamma_{2 i}(\cdot)$ are known smooth positive definite functions, and $\alpha_{i}(\cdot)$ is a known class $\mathcal{K}_{\infty}$ function satisfying $\lim \sup _{s \rightarrow 0+}\left(\alpha_{i}^{-1}\left(s^{2}\right) / s\right) \leq \infty$.

Then a new simple controller is proposed in this case:

$$
\begin{aligned}
u_{i} & =-\Psi_{i} \eta_{i}-\rho_{i}\left(e_{v i}\right) e_{v i}-\gamma_{i} \operatorname{sgn}\left(e_{v i}\right) \\
\dot{\eta}_{i} & =F_{i} \eta_{i}+G_{i} u_{i} .
\end{aligned}
$$

Performing a transformation $\hat{\eta}_{i}=\eta_{i}-\tau_{i}-G_{i} e_{i}$ gives

$$
\left\{\begin{array}{l}
\dot{\bar{z}}_{i}=\bar{f}_{i}\left(\bar{z}_{i}, e_{i}, v, w, \mu\right) \\
\dot{\hat{\eta}}_{i}=F_{i} \hat{\eta}_{i}+F_{i} G_{i} e_{i}-G_{i} \bar{g}_{i}\left(\bar{z}_{i}, e_{i}, v, w, \mu\right) \\
\dot{e}_{i}=\bar{g}_{i}\left(\bar{z}_{i}, e_{i}, v, w, \mu\right)-\Psi_{i} \hat{\eta}_{i}-\Psi_{i} G_{i} e_{i}+u_{i}^{r} .
\end{array}\right.
$$

Denote $\hat{z}_{i}=\operatorname{col}\left(\bar{z}_{i}, \hat{\eta}_{i}\right)$, and the next lemma can be proved in a similar way given in the last subsection.

Lemma 2 Under Assumption 5, for any given positive constant $M$ and for $i=1, \ldots, n$, there is a positive constant $\bar{M}$ and a smooth Lyapunov function $V_{\hat{z}_{i}}(\cdot)$ satisfying $\hat{\alpha}_{1 i}\left(\left\|\hat{z}_{i}\right\|\right) \leq V_{\hat{z}_{i}}\left(\hat{z}_{i}\right) \leq \hat{\alpha}_{2 i}\left(\left\|\hat{z}_{i}\right\|\right)$ for some smooth functions $\hat{\alpha}_{1 i}(\cdot), \hat{\alpha}_{2 i}(\cdot) \in \mathcal{K}_{\infty}$, such that, for all $\left(v(0), \omega_{i}(0), \mu\right) \in \mathbb{R}_{M}^{\hat{n}}, w(t) \in \mathbb{R}_{M}^{n_{w}}$ and $\eta_{i}(0) \in \mathbb{R}_{\bar{M}}^{n_{\eta_{i}}}$,

$$
\left.\dot{V}_{\hat{z}_{i}}\left(\hat{z}_{i}\right)\right|_{(22)} \leq-\left\|\hat{z}_{i}\right\|^{2}+\hat{\gamma}_{1 i}\left(e_{i}\right)+\hat{\gamma}_{2 i}(w)+\hat{\delta}
$$

where $\hat{\gamma}_{1 i}(\cdot), \hat{\gamma}_{2 i}(\cdot)$ are known smooth positive definite functions and $\hat{\delta}$ is a known positive constant.

Then we show how the control (21) solves our problem.

Theorem 2 Under Assumptions 1-3 and 5, our problem can be semi-globally solved by the distributed control (21).

Proof. The proof is similar to that of Theorem 1.

Step 1: We first prove the boundedness of $(\hat{z}, e)$. Based on Assumption 5 and Lemma 2, we apply the changing supply functions technique and obtain that, for any given $M>0$ and any smooth function $\Delta_{i}\left(\hat{z}_{i}\right)$, there is a positive constant $\bar{M}$ and a smooth function $W_{\hat{z}_{i}}\left(\hat{z}_{i}\right)$ satisfying $\hat{\alpha}_{3 i}\left(\left\|\hat{z}_{i}\right\|\right) \leq W_{\hat{z}_{i}}\left(\hat{z}_{i}\right) \leq \hat{\alpha}_{4 i}\left(\left\|\hat{z}_{i}\right\|\right)$ for some smooth functions $\hat{\alpha}_{3 i}(\cdot), \hat{\alpha}_{4 i}(\cdot) \in \mathcal{K}_{\infty}$, such that, for any $\left(v(0), \omega_{i}(0), \mu\right) \in \mathbb{R}_{M}^{\hat{n}}, w(t) \in \mathbb{R}_{M}^{n_{w}}$ and $\eta_{i}(0) \in \mathbb{R}_{\bar{M}}^{n_{\eta_{i}}}$,

$$
\begin{aligned}
\left.\dot{W}_{\hat{z}_{i}}\right|_{(22)} \leq & -\Delta_{i}\left(\hat{z}_{i}\right)\left\|\hat{z}_{i}\right\|^{2} \\
& +\hat{\gamma}_{3 i}\left(e_{i}\right) e_{i}{ }^{2}+\hat{\gamma}_{4 i}(w)\|w\|^{2}+\hat{\delta}_{1}
\end{aligned}
$$

where $\hat{\gamma}_{3 i}(\cdot), \hat{\gamma}_{4 i}(\cdot)$ are known smooth positive functions and $\hat{\delta}_{1}$ is a known positive constant.

Consider a positive definite and radially unbounded function $\bar{V}(\hat{z}, e)=W_{0}(\hat{z})+V_{0}(e)$. For a given $M>0$, by similar arguments in Theorem 1 , there exist known smooth positive functions $\phi_{1 i}, \phi_{2 i}, \tilde{\phi}_{i}$ such that

$$
\begin{array}{r}
\left\|\Upsilon_{3 i}\right\|^{2} \leq \phi_{1 i}\left(\hat{z}_{i}\right)\left\|\hat{z}_{i}\right\|^{2}+\phi_{2 i}\left(e_{i}\right)\left\|e_{i}\right\|^{2} \\
\sum_{i=1}^{n}\left[\hat{\gamma}_{3 i}\left(e_{i}\right)+\phi_{2 i}\left(e_{i}\right)\right] e_{i}{ }^{2} \leq \sum_{i=1}^{n} \tilde{\phi}_{i}\left(e_{v i}\right) e_{v i}^{2}
\end{array}
$$

where $\Upsilon_{3 i} \triangleq \hat{g}_{i}\left(\bar{z}_{i}, e_{i}, v, \mu\right)-\Psi_{i} \hat{\eta}_{i}-\Psi_{i} G_{i} e_{i}$. By (17), (23), (24) and $\gamma_{i}>0$, letting $\Delta_{i}\left(\hat{z}_{i}\right)=-\phi_{1 i}\left(\hat{z}_{i}\right)+1, \rho_{i}\left(e_{v i}\right)=$ $\frac{1}{2}\left(\tilde{\phi}_{i}\left(e_{v i}\right)+4\right)$ yields $\left.\dot{\bar{V}}\right|_{(22)} \leq-\|\hat{z}\|^{2}-\left\|e_{v}\right\|^{2}+\Xi_{3}$, where

$$
\Xi_{3} \triangleq \sum_{i=1}^{n}\left[\hat{\gamma}_{4 i}(w)+\bar{b}_{2}^{2}\right]\|w\|^{2}+n \hat{\delta}_{1}+\sum_{i=1}^{n}\left\|\mathbf{u}_{i}(v, \mu)\right\|^{2} .
$$


Again by similar techniques used in Theorem 4.14 in Khalil (2002), we obtain the uniform boundedness of the trajectory $(\hat{z}, e)$ and a positive constant $\hat{M}$, only depending on $M$ and $\bar{M}$, satisfying $\left\|\Upsilon_{4 i}\right\| \leq \hat{M}$ with $\Upsilon_{4 i} \triangleq \bar{g}_{i}\left(\bar{z}_{i}, e_{i}, v, w, \mu\right)-\Psi_{i} \hat{\eta}_{i}-\Psi_{i} G_{i} e_{i}-\rho_{i}\left(e_{v i}\right) e_{v i}$.

Step 2: Check $e$ by considering the set-valued Lie derivative of $V_{0}(e)=e^{T} H e$. Taking $\gamma_{i} \geq \hat{M}+1$ gives

$$
\begin{aligned}
\left.\dot{V}_{0}\right|_{(22)} & =2 \sum_{i=1}^{n} e_{v i}^{T}\left[\Upsilon_{4 i}-\gamma_{i} \operatorname{sgn}\left(e_{v i}\right)\right] \\
& \leq-2 \sum_{i=1}^{n}\left(\gamma_{i}-\hat{M}\right)\left|e_{v i}\right| \leq-2 \sum_{i=1}^{n}\left|e_{v i}\right| .
\end{aligned}
$$

Thus, $\lim _{t \rightarrow+\infty} e(t)=0$, which implies the conclusion.

\section{Simulations}

To illustrate our control design, we consider a multiagent system with three non-identical followers in the form of high-order FitzHugh-Nagumo dynamics (Xu \& Huang, 2010) as follows:

$$
\left\{\begin{array}{l}
\dot{x}_{1 i}=x_{1 i}-\frac{1}{3} x_{1 i}^{3}-x_{2 i}+x_{3 i}+d_{i}(t)+b_{i} u_{i} \\
\dot{x}_{2 i}=x_{1 i}+c_{1 i}-x_{2 i} \\
\dot{x}_{3 i}=-x_{1 i}+c_{2 i}-x_{3 i}, \quad i=1,2,3
\end{array}\right.
$$

where $c_{1 i}, c_{2 i}$, and $b_{i}$ are positive constants. The local disturbances are generated by (3) with $D_{1}=1+$ $\mu_{1}, S_{1}=0, D_{2}=\left[1+\mu_{2}, 0\right], S_{2}=[0,1 ; 0,0], D_{3}=$ $\left[1+\mu_{3}, 0\right], S_{3}=[0,1 ;-1,0]$. We aim to make $x_{1 i}$ follow a reference $y_{0}=\left(1+\mu_{v}\right) v_{1}$ generated by a leader (Grasman, 1987) with the input $p(t)$ unknown to the followers as follows:

$$
\left\{\begin{array}{l}
\dot{v}_{1}=\epsilon_{0}\left(-2 v_{1}-v_{2}^{3}-v_{2}\right)+p(t) \\
\dot{v}_{2}=v_{1}-v_{2}
\end{array}\right.
$$

where $p(t)=\frac{2 A}{T}\left(t-T\left\lfloor\frac{t}{T}+\frac{1}{2}\right\rfloor\right)(-1)^{\left\lfloor\frac{t}{T}+\frac{1}{2}\right\rfloor}$ is a triangle wave signal with period $2 T$ and amplitude $A$, and $\lfloor x\rfloor$ is the largest integer not greater than $x$. Denote $\mu \triangleq \operatorname{col}\left(\mu_{v}, \mu_{x}, \mu_{1}, \mu_{2}, \mu_{3}\right)$ as the uncertain parameter vector. Figure 1 describes this multi-agent interaction topology with $a_{i j}=1$, and Figure 2(a) depicts the reference trajectory with $v_{1}(0)=0.1$ and $v_{2}(0)=0$.

Without knowing the exact form of $p(t)$, the formulation in Su \& Huang $(2013)$ even with a nonlinear exosystem (Chen \& Huang, 2005) fails to solve this problem for our multi-agent system. Nevertheless, the problem is solvable based on the formulation in Section 2. In fact, Assumption 2 is verified by a Lyapunov function $V=v_{1}^{2}+\frac{\epsilon_{0}}{2} v_{2}^{4}+\epsilon_{0} v_{2}^{2}$. Let $z_{1 i}=x_{2 i}-c_{1 i}, z_{2 i}=x_{3 i}-$ $c_{2 i}, y_{i}=x_{1 i}$, and the plant is of the form (2) satisfying

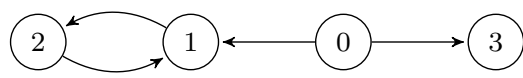

Fig. 1. Interaction graph $\mathcal{G}$ in our example.

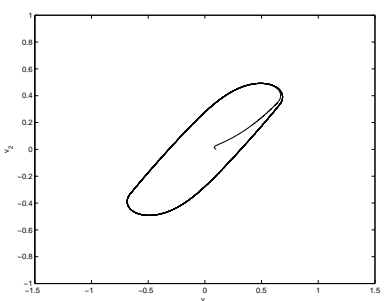

(a)

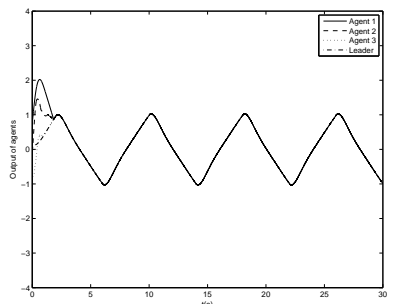

(b)
Fig. 2. Trajectory of the leader and performance of the control law.

Assumption 3 with $\mathbf{z}_{1 i}=\left(1+\mu_{v}\right) v_{2}, \mathbf{z}_{2 i}=-\left(1+\mu_{v}\right) v_{2}$. Assumption 4 also holds with $V_{\bar{z}_{i}}(s)=\frac{1}{2} s^{2}, \alpha_{i}(s)=$ $\frac{1}{2} s^{2}, \gamma_{1 i}(s)=\gamma_{2 i}(s)=s^{2}$. By Theorem 1 , the control with $\rho_{i}(s)=s^{6}+1$ can solve this problem. To reduce the unfavorable chattering in simulations, the sign function in the proposed control (13) can be replaced by a saturation function as follows:

$$
\operatorname{sat}_{\epsilon}(x)= \begin{cases}x / \epsilon, & \text { if }|x| \leq \epsilon \\ \operatorname{sgn}(x / \epsilon), & \text { if }|x|>\epsilon\end{cases}
$$

With selected matrix pairs $\left(F_{i}, G_{i}\right)$, Figure $2(\mathrm{~b})$ shows the performance of the controller with $A=2, T=4$, $\epsilon=10^{-3}, c_{1 i}=c_{2 i}=b_{i}=i$ and $\mu \in[-1,1]^{5}$.

\section{Conclusions}

In this paper, a distributed output regulation problem was formulated for a class of uncertain heterogeneous nonlinear multi-agent systems to deal with local disturbances and an unknown-input leader. Based on changing supply functions and adaptive techniques, distributed control laws incorporating local internal models were constructed. The semi-global and global results were obtained in two different cases.

\section{References}

Cao, Y., \& Ren, W. (2012). Distributed coordinated tracking with reduced interaction via a variable structure approach. IEEE Transactions on Automatic Control, 57(1), 33-48.

Chen, Z., \& Huang, J. (2005). Robust output regulation with nonlinear exosystems. Automatica, 41(8), 14471454.

Cortes, J. (2008). Discontinuous dynamical systems.IEEE Control Systems, 28(3), 36-73.

Ding, Z. (2013). Consensus output regulation of a class of heterogeneous nonlinear systems. IEEE Transactions on Automatic Control, 58(10), 2648-2653. 
Dong, Y., \& Huang, J. (2014). Cooperative global robust output regulation for nonlinear multi-agent systems in output feedback form. Journal of Dynamic Systems, Measurement, and Control, 136(3), 031001.

Godsil, C., \& Royle, G. (2001). Algebraic graph theory. New York: Springer.

Grasman, J. (1987). Asymptotic methods for relaxation oscillations and applications. New York: Springer.

Hong, Y., Hu, J., \& Gao, L. (2006). Tracking control for multi-agent consensus with an active leader and variable topology. Automatica, 42(7), 1177-1182.

Hong, Y., Wang, X., \& Jiang, Z. P. (2013). Distributed output regulation of leader-follower multi-agent systems. International Journal of Robust and Nonlinear Control, 23(1), 48-66.

Huang, J. (2004). Nonlinear output regulation: Theory and applications. Philadelphia: SIAM.

Isidori, A., Marconi, L., \& Serrani, A. (2003). Robust autonomous guidance: An internal model-based approach. London: Springer.

Jiang, Z.-P., \& Hill, D. J. (1999). A robust adaptive backstepping scheme for nonlinear systems with unmodeled dynamics. IEEE Transactions on Automatic Control, 44(9), 1705-1711.

Khalil, H. K. (2002). Nonlinear systems (3rd ed.). New Jersey: Prentice-Hall.

Li, Z., Liu, X., Ren, W., \& Xie, L. (2013). Distributed tracking control for linear multi-agent systems with a leader of bounded unknown input. IEEE Transactions on Automatic Control, 58(2), 518-523.

Liu, K., Xie, G., Ren, W., \& Wang, L. (2013). Consensus for multi-agent systems with inherent nonlinear dynamics under directed topologies. Systems \& Control Letters, 62(2), 152-162.

Praly, L. (2003). Asymptotic stabilization via output feedback for lower triangular systems with output dependent incremental rate. IEEE Transactions on Automatic Control, 48(6), 1103-1108.

Ramos, L. E., Celikovsky, S., \& Kucera, V. (2004). Generalized output regulation problem for a class of nonlinear systems with nonautonomous exosystem. IEEE Transactions on Automatic Control, 49(10), 17371743.

Saberi, A., Stoorvogel, A. A. \& Sannuti P. (2001). On output regulation for linear systems. International Journal of Control, 74(8), 783-810.

Sontag, E., \& Teel, A. (1995). Changing supply functions in input/state stable systems. IEEE Transactions on Automatic Control, 40(8), 1476-1478.

Su, Y., \& Huang, J. (2013). Cooperative adaptive output regulation for a class of nonlinear uncertain multiagent systems with unknown leader. Systems $\&$ Control Letters, 62(6), 461-467.

Tang, Y. (2014). Leader-following coordination problem with an uncertain leader in a multi-agent system. IET Control Theory \& Applications, 8(10), 773-781.

Tang, Y. \& Hong, Y. (2013). Hierarchical distributed control design for multi-agent systems using approximate simulation. Acta Automatica Sinica, 39(6), 868-
874.

Xu, D., \& Huang, J. (2010). Robust adaptive control of a class of nonlinear systems and its applications. IEEE Transactions on Circuits and Systems I: Regular Papers, 57(3), 691-702.

Yang, X., \& Huang, J. (2010). A framework for nonlinear output regulation for time-varying uncertain systems. Proceedings of the 49th IEEE Conference on Decision and Control (pp. 5396-5401). Atlanta, USA. 